\title{
Congenital heart block
}

INSERM

\section{Source}

INSERM. (1999). Orphanet: an online rare disease and orphan drug data base. Congenital heart block. ORPHA:60041

Congenital heart block (CHB) is a rare disorder of atrioventricular conduction,

characterized by absence of conduction of atrial impulses to the ventricles with slower ventricular rhythm (atrioventricular dissociation). CHB can occur in association with immunological evidence of maternal connective disease (autoimmune CHD), fetal structural CHD or can be idiopathic. 